\title{
MCMAS: A model checker for the verification of multi-agent systems ${ }^{\star}$
}

\author{
Alessio Lomuscio*, Hongyang Qu*, Franco Raimondi ${ }^{\dagger}$ \\ ${ }^{*}$ Imperial College London, UK ${ }^{\dagger}$ University College London, UK
}

\section{Overview}

While temporal logic in its various forms has proven essential to reason about reactive systems, agent-based scenarios are typically specified by considering high-level agents attitudes. In particular, specification languages based on epistemic logic [7], or logics for knowledge, have proven useful in a variety of areas including robotics, security protocols, web-services, etc. For example, security specifications involving anonymity [4] are known to be naturally expressible in epistemic formalisms as they explicitly state the lack of different kinds of knowledge of the principals.

More generally, various extensions of temporal logic have been studied in agents and AI contexts to represent properties of autonomous systems. In addition to epistemic operators, at the very core of these approaches is the importance of deontic modalities expressing norms and compliance/violation with respect to previously agreed commitments, and ATL-like modalities expressing cooperation among agents.

While these languages have been long explored and appropriate semantics developed, until recently there has been a remarkable gap in the availability of efficient symbolic model checking toolkits supporting these. In this paper we describe MCMAS, a symbolic model checker specifically tailored to agent-based specifications and scenarios. MCMAS [12] supports specifications based on CTL, epistemic logic (including operators of common and distributed knowledge) [7], Alternating Time Logic [2], and deontic modalities for correctness [16]. The release described in this abstract is a complete rebuild of a preliminary experimental checker [14]. The model input language includes variables and basic types and it implements the semantics of interpreted systems, thereby naturally supporting the modularity present in agent-based systems. MCMAS implements OBDD-based algorithms optimised for interpreted systems and supports fairness, counter-example generation, and interactive execution (both in explicit and symbolic mode). MCMAS has been used in a variety of scenarios including web-services, diagnosis, and security. MCMAS is released under GNU-GPL.

\section{Multi-Agent Systems Formalisms}

Multi-Agent Systems (MAS) formalisms are typically built on extensions of computational tree logic (CTL). For the purposes of this abstract we consider specifications given in the following language $\mathcal{L}$ built from a set of propositional atoms $p \in P$, and a set of agents $i \in A$ ( $G \subseteq A$ denotes a set of agents):

\footnotetext{
* The research described in this paper is partly supported by the European Commission Framework 6 funded project CONTRACT (IST Project Number 034418).
} 
$\phi::=\neg \phi|\phi \wedge \phi|\langle G\rangle\rangle X \phi|\langle\langle G\rangle\rangle F \phi|\langle\langle G\rangle\rangle[\phi U \psi]\left|K_{i} \phi\right| D_{G} \phi\left|C_{G} \phi\right| O_{i} \phi$.

$\mathcal{L}$ extends ATL (hence CTL) by considering epistemic modalities representing "agent $i$ knows $\phi$ " $\left(K_{i} \phi\right)$, "group $G$ has distributed knowledge of $\phi$ " $\left(D_{G} \phi\right)$, "group $G$ has common knowledge of $\phi$ " $\left(C_{G} \phi\right)$, and deontic modalities encoding "whenever agent $i$ is working correctly $\phi$ holds" $\left(O_{i} \phi\right)$. The ATL modalities above are read as customary: $\langle\langle G\rangle\rangle X \phi$ stands for "group $G$ can enforce $\phi$ at the next step" and $\langle\langle G\rangle\rangle F \phi$ stands for "group $G$ can enforce $\phi$ at some point in the future". As standard, CTL modalities for $A G, A U, A X$ and their existential counterparts may be derived from the ATL modalities, and, similarly, epistemic modalities for $E_{G}$ ("everyone in $G$ knows") may be rewritten as a conjunction of appropriate $K_{i}, i \in G$, formulas. The specification language above is very rich as it includes AI-based modalities representing various notions of knowledge [7], deontic conditions [16], ATL-style modalities for cooperation [2], as well as standard CTL.

A computationally grounded semantics for the family of MAS formalisms above (in the sense of [19], i.e., one in which the interpretation to all modalities is defined in terms of the computational states of the system) can be given by suitably extending interpreted systems. Interpreted systems [7], originally proposed for linear time only, are an agentbased semantics where the components, or agents, are defined by a set of possible local states, a set of actions that they may perform according to their local protocol, and transition functions returning the target local state given the current local state and the set of actions performed by all agents. An environment (described similarly to an agent) is also modelled as part of the system. ATL and CTL modalities are interpreted on the induced temporal relation given by the protocols and transition functions [15], the epistemic modalities are defined on the equivalence relations built on the equality of local states [7], and the deontic modalities are interpreted on "green states", i.e., subsets of local states representing states of locally correct behaviour for the agent in question. Specifically, satisfaction for the epistemic modalities is defined by $(I S, s) \models K_{i} \phi$ iff for all $s^{\prime} \in S$ we have that $s \sim_{i} s^{\prime}$ implies $\left(I S, s^{\prime}\right) \models \phi$, where $I S$ is an interpreted system, $s, s^{\prime}$ reachable global states, and $\sim_{i}$ is defined on the local equality of global states, i.e., $s \sim_{i} s^{\prime}$ iff $l_{i}(s)=l_{i}\left(s^{\prime}\right)$ where $l_{i}$ is the function returning the local state of agent $i$ in a given global state. Satisfaction for common knowledge is defined by $(I S, s) \models C_{G} \phi$ iff for all $s^{\prime} \in S$ we have that $s \sim^{*} s^{\prime}$ implies $\left(I S, s^{\prime}\right) \models \phi$, where $\sim^{*}$ is the reflexive and transitive closure of the union of the relations $\sim_{i}, i \in G$. We refer to the user manual available from [12] for satisfaction of distributed knowledge $D_{G}$ and correctness modalities $O_{i}$, as well as more details and examples.

The language $\mathcal{L}$ has been used to specify a wide range of scenarios in application areas such as web-services, security, and communication protocols. For example, in a communication protocol we can use $E F\left(K_{\text {sender }}\left(K_{\text {receiver }}(\right.\right.$ bit $\left.\left.=0)\right)\right)$ to specify that at some point in the future the sender will know that the receiver knows that the bit being sent is equal to 0 ; in a game-based setting, we can write $A G O_{p_{1}}\left\langle\left\langle p_{1}, p_{2}\right\rangle\right\rangle X\left(p_{1} \_p_{2} w i n\right)$ to represent that it is always the case that, as long as player 1 is functioning correctly, player 1 and player 2 can together force a win at any step.

The complexity of the model checking problem of $\mathcal{L}$ against compact representations (e.g., via reactive modules, or ISPL modules as below) is given by its more expensive fragment (ATL) and so it is EXPTIME-complete [9]. Note, however, that 
the problem of checking its temporal-epistemic-deontic fragment is only PSPACEcomplete [13], i.e., the same as CTL [11].

\section{The MCMAS toolkit}

MCMAS is implemented in $\mathrm{C}++$ and compiled for all major platforms. It exploits the CUDD [18] library for BDD operations. MCMAS implements standard algorithms for CTL and ATL [3, 2], and dedicated BDD-based algorithms for the epistemic and deontic operators [17], in particular, the algorithms for satisfaction for $K_{i}$ and $C_{G}$ are sketched in Algorithm 1 ( $S$ represents the set of reachable states).

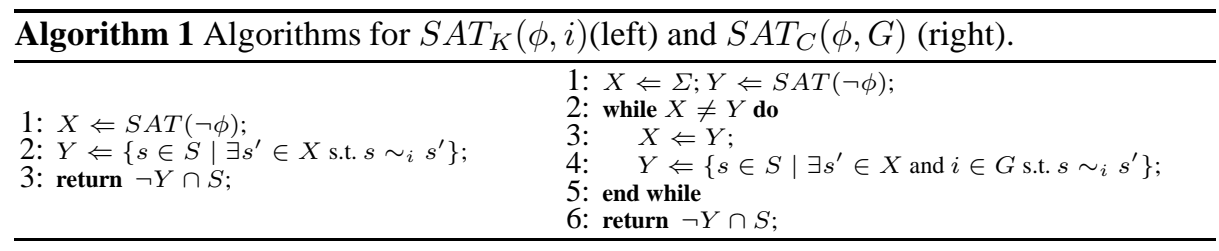

A number of optimisations are implemented in MCMAS in an attempt to minimise the memory consumption and verification time. For example, the checker does not build a single OBDD for the global transition relation, but performs any required operation against the local evolutions. Also, MCMAS does not compute the union of equivalence relations $\sim_{i}$ in Algorithm 1 when checking common knowledge, but instead repeatedly operates on all $\sim_{i}$. MCMAS provides counterexamples and witnesses for a wide range of formulas including epistemic modalities thereby giving guidance to the user. The algorithm used to return witnesses and counterexamples is novel and inspired by the tree-like construction of [5].

MCMAS takes ISPL (Interpreted Systems Programming Language) descriptions as input. An ISPL file fully describes a multi-agent system (both the agents and the environment), and it closely follows the framework of interpreted systems described above. We refer to the user manual for examples and usage. Essentially, an ISPL agent is described by giving the agents' possible local states, their actions, protocols, and local evolution functions. Local states are defined by using variables of type Boolean, integer, and enumeration. An optional section RedStates permits the definition of non-green states by means of any Boolean formula on the variables of the local states to interpret the correctness modalities $O_{i}$. The local transition function is given as a set of evolution items of the form A if C, where $\mathrm{C}$ is a Boolean condition over local variables, global variables (see below), and actions by the agents and the environment, and $A$ is a set of assignments on the agent's local variables. All variables not present in A remain constant in the local transition. Any enabling condition and synchronisation among the agents is specified in C; note also that any non-deterministic behaviour may be specified by using several evolution items. Compared to that of an agent, an environment definition may have additional features, including the definition of global variables observable to some or all agents. Table 1 shows an example of a self-explanatory ISPL file for the Train/Gate/Controller scenario with two trains.

An ISPL file also contains sections for the definition of the initial states (given by Boolean conditions on the agents' local variables), any fairness constraints, groups 
On a Linux x86_64 machine with Intel Core 2 Duo 2.2GHz and 4GB memory, we tested the protocol (code on the website) against two temporal epistemic specifications: $A G\left(\left(\right.\right.$ odd $\wedge \neg$ payer $\left._{1}\right) \rightarrow\left(\left(K_{\text {cryptographer }} \bigvee_{i=2}^{n}\right.\right.$ payer $\left._{i}\right) \wedge\left(\bigwedge_{i=2}^{n} \neg K_{\text {cryptographer }_{1}}\right.$ payer $\left.\left.\left._{i}\right)\right)\right)$, $A G\left(\right.$ even $\rightarrow C_{\left\{\text {cryptographer }_{1}, \ldots, \text { cryptographer }_{n}\right\}} \neg\left(\bigvee_{i=1}^{n}\right.$ payer $\left.\left._{i}\right)\right)$. We checked the second formula specifically to evaluate the performance of the tool against common knowledge.

Table 2. Verification results for the dinning cryptographers protocol.

\begin{tabular}{|c|c|c|c|c|c|c|}
\hline$n$ crypts & possible & reachable & \multicolumn{2}{|c|}{ knowledge } & \multicolumn{2}{c|}{ common knowledge } \\
\cline { 4 - 7 } & states & states & bdd memory (MB) & time (s) & bdd memory (MB) & time (s) \\
\hline 10 & $1.86 \times 10^{11}$ & 33792 & 12.5 & 1 & 12.5 & 1 \\
\hline 11 & $2.23 \times 10^{12}$ & 73728 & 12.4 & 3 & 12.6 & 2 \\
\hline 12 & $2.67 \times 10^{13}$ & 159744 & 12.8 & 4 & 12.9 & 4 \\
\hline 13 & $3.21 \times 10^{14}$ & 344064 & 28.2 & 23 & 28.4 & 23 \\
\hline 14 & $3.85 \times 10^{15}$ & 737280 & 15.8 & 14 & 16.1 & 13 \\
\hline 15 & $4.62 \times 10^{16}$ & $1.57 \times 106$ & 17.1 & 24 & 18.0 & 24 \\
\hline 16 & $5.55 \times 10^{17}$ & $3.34 \times 106$ & 42.3 & 149 & 42.3 & 150 \\
\hline 17 & $6.66 \times 10^{18}$ & $7.07 \times 106$ & 60.0 & 612 & 60.0 & 612 \\
\hline 18 & $7.99 \times 10^{19}$ & $1.49 \times 107$ & 222.8 & 2959 & 222.8 & 2959 \\
\hline
\end{tabular}

The table reports the results for different numbers of cryptographers, indicated in the first column. The size of the state space (equal to $3 \times 12^{n}$ ) is reported in the second column, and the third reports the number of actual reachable states in the corresponding model. Memory usage and time required for the verification of the two formulas follow in the last four columns respectively.

A direct efficiency comparison with other toolkits is problematic. Apart from the different input languages, other tools with overlapping functionalities support different variable types making any comparison difficult. In terms of pure size of the model, we found that MCMAS can explore the full state space of models whose size is approximately two orders of magnitude larger than most examples available for temporalepistemic model checkers $[8,6,20]$, and comparable to the size of the models analysed with BDD-based temporal-only model checkers such as NuSMV.

As mentioned in the introduction, MCMAS is a complete reimplementation of the original proof-of-concept described in [14]. Compared to the original prototype, the current version is several orders of magnitude faster. This is due to improved algorithms for the verification of epistemic and ATL modalities, and the computation of the reachable state space. Additionally, the revised input language now enables the user to write code that naturally generates smaller models, e.g., by using globally observable variables in the environment. Several functionalities, e.g., counterexample generation, witnesses, fairness, a fully-fledged GUI, etc., are also now included.

From the point of view of supported functionalities, MCMAS is the only checker we are aware of that supports the specification language $\mathcal{L}$ described above. Epistemic modalities are also treated in [8] although not via an observation-based semantics as here and not with the CUDD package. BMC based approaches for epistemic modalities have also been presented [6]: a comparison with [6] reveals the known advantages and 
disadvantages of BDD vs SAT-based approaches. Finally, ATL is of course supported by MOCHA [1]. However, MOCHA is an on-the-fly checker tailored to assume/guarantee analysis, whose efficiency crucially depends on the learning of successful decompositions and assumptions for the scenario under analysis.

\section{References}

1. R. Alur, T. Henzinger, F. Mang, S. Qadeer, S. Rajamani, and S. Tasiran. MOCHA: Modularity in model checking. In Proceedings of the 10th International Conference on Computer Aided Verification (CAV'98), volume 1427 of LNCS, pages 521-525. Springer-Verlag, 1998.

2. R. Alur, T. A. Henzinger, and O. Kupferman. Alternating-time temporal logic. Journal of the ACM, 49(5):672-713, 2002.

3. J. R. Burch, E. M. Clarke, K. L. McMillan, D. L. Dill, and L. J. Hwang. Symbolic model checking: $10^{20}$ states and beyond. Information and Computation, 98(2):142-170, 1990.

4. D. Chaum. The dining cryptographers problem: Unconditional sender and recipient untraceability. Journal of Cryptology, 1(1):65-75, 1988.

5. E. Clarke, Y. Lu, S. Jha, and H. Veith. Tree-like counterexamples in model checking. In the $17^{\text {th }}$ IEEE Symposium on Logic in Computer Science. IEEE Computer Society, 2002.

6. P. Dembiński, A. Janowska, P. Janowski, W. Penczek, A. Pólrola, M. Szreter, B. Woźna, and A. Zbrzezny. VerICs: A tool for verifying Timed Automata and Estelle specifications. In Proceedings of TACAS'03, volume 2619 of LNCS, pages 278-283. Springer-Verlag, 2003.

7. R. Fagin, J. Y. Halpern, Y. Moses, and M. Y. Vardi. Reasoning about Knowledge. MIT Press, Cambridge, 1995.

8. P. Gammie and R. van der Meyden. MCK: Model checking the logic of knowledge. In Proceedings of CAV'04, volume 3114 of LNCS, pages 479-483. Springer-Verlag, 2004.

9. W. van der Hoek, A. Lomuscio, and M. Wooldridge. On the complexity of practical atl model checking knowledge, strategies, and games in multi-agent systems. In Proceedings of AAMAS'06, pages 946-947. ACM Press, 2006.

10. M. Kacprzak, A. Lomuscio, A. Niewiadomski, W. Penczek, F. Raimondi, and M. Szreter. Comparing BDD and SAT based techniques for model checking Chaum's dining cryptographers protocol. Fundamenta Informaticae, 63(2,3):221-240, 2006.

11. O. Kupferman, M. Y. Vardi, and P. Wolper. An automata-theoretic approach to branchingtime model checking. Journal of the ACM, 47(2):312-360, 2000.

12. A. Lomuscio, H. Qu, and F. Raimondi. MCMAS. http://www-lai.doc.ic.ac.uk/mcmas/.

13. A. Lomuscio and F. Raimondi. The complexity of model checking concurrent programs against CTLK specifications. In Proceedings of the $5^{t} h$ international joint conference on Autonomous agents and multiagent systems (AAMAS'06), pages 548-550. ACM Press, 2006.

14. A. Lomuscio and F. Raimondi. MCMAS: A model checker for multi-agent systems. In Proceedings of TACAS 2006, volume 3920, pages 450-454. Springer Verlag, 2006.

15. A. Lomuscio and F. Raimondi. Model checking knowledge, strategies, and games in multiagent systems. In Proceedings of AAMAS'06, pages 161-168. ACM Press, 2006.

16. A. Lomuscio and M. Sergot. Deontic interpreted systems. Studia Logica, 75(1):63-92, 2003.

17. F. Raimondi and A. Lomuscio. Automatic verification of multi-agent systems by model checking via OBDDs. Journal of Applied Logic, 5(2):235-251, 2005.

18. F. Somenzi. CUDD: CU decision diagram package - release 2.4.1. http://vlsi.colorado.edu/ fabio/CUDD/cuddIntro.html, 2005.

19. M. Wooldridge. Computationally grounded theories of agency. In Proceedings of ICMAS, International Conference of Multi-Agent Systems, pages 13-22. IEEE Press, 2000.

20. M. Wooldridge, M. Fisher, M. Huget, and S. Parsons. Model checking multiagent systems with MABLE. In Proceedings of AAMAS'02, pages 952-959, 2002. 